Author manuscript of: Van den Bos, E., Van Duijvenvoorde, A. C. K., \& Westenberg, P. M. (2016).

Effects of adolescent socio-cognitive development on the cortisol response to social evaluation.

Developmental Psychology, 52, 1151-1163. http://dx.doi.org/10.1037/dev0000133

(C) 2016 American Psychological Association

This article may not exactly replicate the authoritative document published in the APA journal. It is not the copy of record. 


\section{Effects of Adolescent Socio-Cognitive Development on the Cortisol Response to Social}

\section{Evaluation}

Esther van den Bos, Anna C. K. van Duijvenvoorde, and P. Michiel Westenberg Institute of Psychology, Leiden University, the Netherlands

Author note

We thank Sindy Sumter, Anne Miers, Laura Compier-de Block, Ellen Middag, Manja Koenders and Masha Takens for their assistance in data collection and Senem Fincan and Jiska Koster for scoring the recursive thinking test.

Correspondence concerning this article should be addressed to Esther van den Bos, Leiden University, Developmental Psychology Unit, PO Box 9555, 2300 RB LEIDEN, The Netherlands, phone: +31 71527 6868, fax: +31 71527 3619, e-mail:

bosejvanden@fsw.leidenuniv.nl 


\begin{abstract}
Adolescents become increasingly sensitive to social evaluation. Some previous studies have related this change to pubertal development. The present longitudinal study examined the role of socio-cognitive development. We investigated whether or not the transition to recursive thinking, the ability to think about (others') thoughts, would be associated with changes in the magnitude and timing of the cortisol response to social evaluation. Salivary cortisol was obtained during the Leiden Public Speaking Task. The task was administered twice with a two-year interval to 221 participants, aged 9-17 years at Time 1. The area under the curve was computed to assess the magnitude of the overall cortisol response. Two difference scores, reflecting speech anticipation and speech delivery, were computed to assess the timing of the cortisol response. Recursive thinking was measured with a cartoon description task. Regression analyses with clustered bootstrap controlling for pubertal development, age and general cognitive functioning showed that the transition to recursive thinking predicted an increase in the cortisol response to speech anticipation, but was unrelated to the magnitude of the overall cortisol response. This is in line with the view that increasing sensitivity to social evaluation in adolescence is mainly due to the effects of pubertal hormones on affective regions of the brain. Socio-cognitive development affected the timing rather than the magnitude of the cortisol response. The results suggest that recursive thinking enables earlier realization of social-evaluative threat.
\end{abstract}

Keywords: adolescence, social evaluation, salivary cortisol response, recursive thinking, socio-cognitive development 


\section{Effects of Adolescent Socio-Cognitive Development on the Cortisol Response to Social Evaluation}

Adolescents show an increasing sensitivity to social-evaluative threat. This threat "occurs when an important aspect of the self-identity is or could be negatively judged by others" (Dickerson \& Kemeny, 2004, p. 358). The magnitude of the cortisol response and other biological stress responses to conditions of social-evaluative threat has been shown to increase across adolescence (e.g. Gunnar, Wewerka, Frenn, Long, \& Griggs, 2009; Gunther Moor, Bos, Crone, \& Van der Molen, 2014; Silk et al., 2012; Somerville, Jones, Ruberry, Dyke, Glover, \& Casey, 2014; Stroud et al., 2009; Van den Bos, de Rooij, Miers, Bokhorst, \& Westenberg, 2014). The adolescent-bound increase in sensitivity to social-evaluative threat has also been found in studies using self-report questionnaires (e.g. Weems \& Costa, 2005; Westenberg, Drewes, Goedhart, Siebelink \& Treffers, 2004). A few studies have investigated the relation between the magnitude of responses to social-evaluative threat and pubertal development (e.g. Gunnar, Wewerka et al., 2009; Stroud et al., 2009; Van den Bos et al., 2014). However, other adolescent developments may also play a role. The present study investigated whether the magnitude and also the timing of the cortisol response to social evaluation are associated with socio-cognitive development during adolescence.

\section{Sensitivity to Social-evaluative Threat in Adolescence}

Social-evaluative threat has been shown to trigger a response of the hypothalamicpituitary-adrenal (HPA-axis) in adults, adolescents and children (for reviews see Dickerson \& Kemeny, 2004; Gunnar, Talge, \& Herrera, 2009). Despite its occurrence across a wide age range, however, several studies have demonstrated that the magnitude of the cortisol response to social evaluation increases during adolescence (Gunnar, Wewerka et al., 2009; KlimesDougan, Hastings, Granger, Usher, \& Zahn-Waxler, 2001; Stroud et al., 2009; Van den Bos et al., 2014). 
The adolescent increase in the magnitude of the cortisol response is in line with increases in other neuroendocrine, physiological and neural responses to instances of social evaluation. Studies using social-evaluative stress tasks, such as public speaking, have also demonstrated age-related increases in the salivary alpha-amylase response (Stroud et al., 2009; Sumter et al., 2010; Van den Bos et al., 2014), although mixed results have been reported for cardiovascular responses (Gunnar, Wewerka et al., 2009; Hollenstein, McNeely, Eastabrook, MacKey, \& Flynn, 2012; Stroud et al., 2009). Experiments in which participants were explicitly informed that they were accepted or rejected by peers provided evidence for heightened physiological responding to rejection. That is, adolescents (and adolescent girls in particular) showed more heart rate slowing than children in response to rejection feedback (Gunther Moor et al., 2014). Similarly, the pupillary response to rejection in a virtual chatroom increased with age in a sample of 9 to 17 year-olds (Silk et al., 2012). Age-related increases in sensitivity to social evaluation were also observed in passive social evaluation. That is, the simple notification of being watched by a peer while lying in an fMRI scanner resulted in higher reports of self-conscious emotions, larger skin conductance responses and more activation of the medial prefrontal cortex ( $\mathrm{mPFC}$ ) in adolescents than in children (Somerville et al., 2014).

Further evidence for an increase in sensitivity to social evaluation during adolescence is provided by developmental research on fears and worries experienced in daily life. Studies on the normative development of fears have shown that the relative frequency of social fears compared to other fears increases from middle childhood to adolescence, with social fears becoming predominant in adolescence (Weems \& Costa, 2005; Westenberg, Gullone, Bokhorst, Heyne, \& King, 2007; Westenberg, Siebelink, Warmenhoven, \& Treffers, 1999). Similarly, the predominant topic of worry reported by children shifted from physical well- 
being in 5- and 6-year-olds to behavioral competence in 8- and 9-year-olds and to behavioral competence and social evaluation in 11 and 12-year-olds (Vasey, Crnic, \& Carter, 1994).

In addition to studies demonstrating that adolescents come to respond more strongly to social-evaluative threat, a few studies have indicated that adolescents also start to respond earlier. That is, the timing of the cortisol response appears to occur earlier during situations eliciting a social-evaluative threat. Sumter, Bokhorst, Miers, Van Pelt, and Westenberg (2010) distinguished between the cortisol response in anticipation of public speaking and the cortisol response to actually delivering a speech. They found that the anticipatory cortisol response in particular was larger in older adolescents. Stroud et al. (2009) reported that adolescents showed heightened cortisol levels just before a social interaction task, while neither children nor adolescents showed a further increase in response to the task itself. Finally, age-related increases in activation of the $\mathrm{mPFC}$ and the striatum were observed while participants were waiting for acceptance feedback after having expressed the expectation to be liked (Gunther Moor, Van Leijenhorst, Rombouts, Crone, \& Van der Molen, 2010).

\section{Underlying Developments}

In the neurodevelopmental literature, the increase in sensitivity to social-evaluative threat has been proposed to result from structural and functional changes in subcortical (limbic) regions and the PFC (e.g. Burnett, Sebastian, Cohen Kadosh, \& Blakemore, 2011; Haller, Cohen Kadosh, \& Lau, 2014; Nelson, Leibenluft, McClure, \& Pine, 2005; Somerville, 2013). In their model of the social information processing network, Nelson et al. (2005) distinguish between an affective node (mainly limbic regions), which determines the emotional value of stimuli, and a cognitive-regulatory node (PFC), which is involved in Theory of Mind (ToM) operations, inhibition and goal-directed behavior. Changes in the affective node occur under the influence of gonadal hormones, whereas changes in the cognitive-regulatory node are less related to pubertal hormones and continue throughout 
adolescence. The increase in sensitivity to social evaluation has mainly been ascribed to hormonal sensitization of the affective node and delayed maturation of (connections with) the cognitive-regulatory node (Burnett et al., 2011; Haller et al., 2014; Nelson et al., 2005; Somerville, 2013).

Sensitization of the affective node by pubertal hormones has received most attention in research exploring the mechanisms behind the increasing magnitude of the cortisol response to social-evaluative threat in adolescence. Three cross-sectional studies found that the increase in the cortisol response to a public speaking task was related to both age and pubertal development (Gunnar, Wewerka et al., 2009; Stroud et al., 2009; Sumter et al., 2010). A longitudinal study showed that the overall cortisol response was more strongly related to pubertal development than to age (Van den Bos et al., 2014).

Up to now, no study has investigated whether development of the cortisol response to social-evaluative threat is related to maturation of the cognitive-regulatory node. The cognitive-regulatory node is involved in ToM and executive functions, which are distinct but related concepts. Inhibition, working memory and cognitive flexibility can be considered basic executive functions, from which higher order functions such as reasoning are built (Diamond, 2013). In most theoretical accounts, ToM operations make use of basic executive functions, but cannot be reduced to them (see Blakemore \& Mills, 2014, for a review). For example, individual differences in the understanding of mental states could not be explained by individual differences in working memory (Vetter, Leipold, Kliegel, Phillips, \& Altgassen, 2013).

Continued development throughout adolescence has been demonstrated both for executive functions (e.g. Diamond, 2013; Huizinga, Dolan, \& Van der Molen, 2006) and for aspects of ToM (Choudhury, Blakemore, \& Charman, 2006; Dumontheil, Apperly, \& Blakemore, 2010; Müller \& Overton, 2010; Van den Bos, De Rooij, Sumter, \& Westenberg, 
2016; Vetter et al., 2013). Nevertheless, these developments may have differential effects on the cortisol response to social-evaluative stress tasks. Improvements in executive functions would presumably make the task of giving a speech or doing mental arithmetic in front of an audience more manageable and hence less stressful. This would be in line with the suggestion that maturation of the cognitive-regulatory node results in a decrease of sensitivity to socialevaluative threat toward adulthood (Burnett et al., 2011; Nelson et al., 2005). In contrast, increasing automaticity of ToM operations has been suggested to contribute to the increase in sensitivity to social evaluation (Haller et al., 2014). Previous studies focusing on the effects of pubertal development have not ruled out this possibility. Moreover, increasing automaticity of ToM operations may enable adolescents to imagine social-evaluative threat before it arises. This may contribute to the findings of age-related increases in anticipatory responses to social-evaluative threat (Gunter Moor et al., 2010; Stroud et al., 2009; Sumter et al., 2010). The present study investigated the possible contributions of a specific aspect of ToM: recursive thinking.

The concept of recursive thinking, or thinking about thinking, was introduced by Miller, Kessel and Flavell (1970). They proposed that it develops in four steps. The first step is thinking about contiguous people (e.g. someone is thinking about John and Jane). The second step is thinking about action between people (e.g. someone is thinking that John is talking to Jane). The third step is thinking about thinking: one-loop recursive thinking (e.g. someone is thinking that John is thinking about Jane). The fourth step is two-loop recursive thinking: thinking about thinking about thinking (e.g. someone is thinking that John is thinking that Jane is thinking about him). While mastery of the first two levels (as assessed with a cartoon description task) is achieved in childhood (Eliot, Lovell, Dayton, \& McGrady, 1979; Landry \& Lyons-Ruth, 1980; Miller et al., 1970; Oppenheimer, 1986; Veith, 1980), 
recent studies have shown that the development of recursive thinking continues during adolescence (Müller \& Overton, 2010; Van den Bos et al., 2016).

As noted by Veith (1980) the ability to think about thinking is a prerequisite for considering what others think about oneself. When children or adolescents shift from nonrecursive thinking to recursive thinking, they gain the ability to think about (other people's) thoughts and become aware that other people think about and evaluate them. Bokhorst, Westenberg, Oosterlaan and Heyne (2008) suggested that this realization may be related to the emergence of a distinct fear of social evaluation in late childhood and adolescence. Likewise, the transition may affect stress responses to social-evaluative situations, such as public speaking. Children at recursive levels of thinking may immediately recognize, or even foresee, that a situation involves social evaluation, whereas children at non-recursive levels of thinking may not realize its social-evaluative nature until they receive some kind of feedback. Therefore, the former may have an earlier onset of the cortisol response than the latter.

\section{Present Study}

Two research questions were addressed in the present study. The first was whether or not the transition from non-recursive thinking to recursive thinking contributed to the adolescent increase in magnitude of the overall cortisol response. The second was whether or not the transition from non-recursive thinking to recursive thinking was associated with an earlier onset of the cortisol response, indicated by a larger response to speech anticipation and a smaller response to speech delivery.

The research questions were investigated using data from a comprehensive longitudinal study on Social Anxiety and Normal Development (SAND). The SAND study included two administrations of the Leiden Public Speaking Task (LPST; Westenberg et al., 2009) over a two-year interval and concurrent assessments of pubertal development and recursive thinking. Two previous longitudinal studies were based on data from the same 
sample. One investigated whether the development of recursive thinking levels off in early adolescence or continues thereafter, demonstrating that the total recursive thinking score, computed over all types of items, increased linearly throughout adolescence (Van den Bos et al., 2016). The other study aimed to disentangle the effects of age and pubertal development on neuroendocrine responses to the LPST (Van den Bos, et al., 2014). The results showed that the overall cortisol and salivary alpha-amylase responses were more strongly related to pubertal development than to age. The overall responses were larger for more mature participants and for participants who reported more pubertal development over the two-year interval. In addition, a puberty-related shift toward anticipation was observed for the cortisol response: more mature participants already reached their peak levels before the actual speech.

The present study expands on these previous studies by investigating whether a specific step in the development of recursive thinking - the transition from non-recursive to recursive thinking - is related to development of the cortisol response to social evaluation and whether such a relation is independent from pubertal development, age and improvements in general cognitive functioning. We identified participants who underwent the transition from non-recursive thinking to recursive thinking during the two-year interval and investigated how the transition affected the overall cortisol response to the LPST as well as the responses to speech anticipation and speech delivery.

\section{Method}

\section{Participants}

The data used in the present study are part of the Social Anxiety and Normal Development (SAND) study. The aims of the SAND-study were a.) to chart the development of social anxiety as well as normative physical and socio-cognitive developments in a community sample of adolescents and b.) to investigate how social anxiety and stress responses to public speaking are related to b.1.) individual difference variables (e.g. Miers, 
Blöte, De Rooij, Bokhorst, \& Westenberg, 2013) and b.2.) normative developments in the physical (e.g. Van den Bos et al., 2014) and socio-cognitive domains. The SAND-study was approved by the Leiden University Medical Ethical Committee and carried out in accordance with the Declaration of Helsinki. Parents provided active consent; written assent was obtained from participants themselves.

Participants were recruited through two primary schools and one secondary school in Leiden, a middle-sized city in the Netherlands. The majority of participants were of Dutch origin: $93.4 \%$ of the participants and $87.4 \%$ of their parents was born in the Netherlands. Eighty-three percent of the participants lived with both biological parents. Participants' parents were relatively well educated: $53 \%$ had completed higher vocational education or graduated from university. The sample included 126 primary school children and 173 adolescents from all educational streams in the Dutch school system (prevocational: $11.0 \%$, first year senior general or pre-university: $20.2 \%$, senior general: $33.5 \%$, pre-university: 35.3\%). Children and adolescents with severe psychological problems or physical illness were excluded from participation. If such problems had been registered at school, students were not invited to participate. To check whether there were any individuals with conditions unknown to the school, participants completed a health and medication history questionnaire probing for treatment by a mental health professional as well as any physical complaints.

The SAND-study had a cohort-sequential design. Data were collected in four waves, with the first wave being fielded in 2006-2007. The recursive thinking test and the LPST were administered in Wave 1 and Wave 3. For clarity, these data collection points will be referred to as Time 1 and Time 2 in the present study. At Time 1, there were 299 participants: 154 males $(51.5 \%)$ and 145 females $(48.5 \%)$. Their ages ranged from 8 to 17 years $(M=13.2, S D$ $=2.3$ ). Table 1 shows the number of participants per grade level and their mean age. At Time 2, two years later, 222 participants returned $(51.4 \%$ male, mean age $=15.0, S D=2.2)$. The 
attrition rate was $25.8 \%$. There was no difference in the distribution of gender $\left(\chi^{2}(1)=.008, p\right.$ $=.928)$ or level of recursive thinking $\left(\chi^{2}(3)=5.906, p=.116\right)$ between those who continued to participate at Time 2 and those who did not. Likewise, there was no difference in mean age $(t(297)<1)$, mean score on the Pubertal Development Scale $(t(286)<1)$, cortisol response (Area Under the Curve with respect to increase: $t(232)=-1.045, p=.297$ ), or mean score on the Social Anxiety Scale $(t(294)=1.123, p=.262)$. This is in line with other data from the SAND-study indicating that attrition over all four waves of data collection was neither related to social anxiety nor to predictors of social anxiety (Miers et al., 2013).

\section{Procedure}

The Leiden Public Speaking Task. The LPST has specifically been designed for longitudinal studies: to enhance reproducibility, participants are informed a week beforehand that they will have to give a speech on a certain topic. The task was modeled on a classroom presentation, with which participants of all ages have experience. Participants deliver their speech in front of a projection screen displaying a life-size audience of age peers and a female teacher, who behave neutrally. They are informed that the audience is prerecorded and that their performance will be recorded and evaluated by peers at a later date (see e.g. Blöte, Bokhorst, Miers, \& Westenberg, 2012, for a report of these evaluations). Making a permanent recording of one's performance has been shown to create a condition of social-evaluative threat and elicit a cortisol response (Dickerson \& Kemeny, 2004). The present situation of ambiguous rather than negative social evaluation may be particularly suitable to reveal developmental differences in sensitivity to social evaluation. As participants have no direct control over the way in which their performance will be evaluated, the LPST combines the two characteristics of laboratory procedures that most consistently trigger a response by the HPA-axis: social-evaluative threat and uncontrollability (Dickerson \& Kemeney, 2004). Previous research has demonstrated a mean cortisol response to the LPST of $2.28 \mathrm{nmol} / \mathrm{l}$ in 
12-15 year-olds (Westenberg et al., 2009). This represents a 44\% increase over resting levels, which is comparable with the cortisol response observed in other studies with an adolescent sample (see Gunnar, Talge et al., 2009, for a review). Because participants are fully informed about the upcoming task, the LPST allows for distinguishing between the effects of speech anticipation and speech delivery.

At both Time 1 and Time 2, participants were invited to the lab twice: once for a presession and once for the public speaking session, one week later. In the pre-session, several self-report questionnaires, cognitive tests —including the recursive thinking test— and a sentence completion test measuring psychosocial development were administered. Participants were tested individually and an (MP3) audio recording was made during the recursive thinking test. The pre-session also served to familiarize participants with the lab and inform them about the public speaking task. They received instructions to prepare a speech on movies they liked or disliked, in the same way as they would for a presentation at school. They were also instructed to refrain from exercising, smoking, eating and drinking caffeinated beverages, dairy products and alcohol one hour before the start of the public speaking session.

The actual public speaking task consisted of seven parts. First, participants watched a nature video while seated $(20 \mathrm{~min})$ and while standing $(5 \mathrm{~min})$. Then, participants received instructions, reminding them of the social-evaluative nature of the task ( 3 min) and they were allowed to rehearse their presentation $(5 \mathrm{~min})$. Subsequently the videotape was started and participants watched the audience enter, after which they delivered their speech ( $5 \mathrm{~min})$. Finally, there was a post-task recovery period (30 min) during which participants completed assessments and watched another 10 minutes of the nature video. All sessions started at 2:15 p.m. to minimize diurnal effects. Full details of the task are provided by Westenberg et al. (2009).

\section{Measures}


Recursive thinking test. An adapted version of the cartoon description task developed by Miller et al. (1970) was used. The cartoon description task involves presenting participants with cartoons that contain thought clouds, speech bubbles and up to four different characters. In each cartoon, the main character is depicted with a thought cloud over his or her head, in which all other elements are embedded. The participant has to describe what the main character is thinking. In the current version, adapted for use with adolescents, contiguity items, which are mastered by second grade elementary school (Miller et al., 1970; Müller \& Overton, 2010; Oppenheimer, 1986), are omitted and two additional two-loop recursion items are presented. Reliability of the adapted version is good and discriminant validity with verbal intelligence could be demonstrated (Van den Bos et al., 2016).

Participants had to describe 14 different cartoons: 3 action items (items 1-3), 5 oneloop recursion items (items 4-8) and 6 two-loop recursion items (items 9-14; see Appendix). The materials were newly created professional drawings (see Figure A1 for an example), printed on A4-sized laminated paper. The experimenter first made sure that the participant understood the basic elements of the cartoons. Clouds with smooth outlines represented talking; clouds with scalloped outlines represented thinking. The characters in the cartoons were one boy, one girl, one man and one woman. Male participants were presented with cartoons in which the boy was the main character and female participants were presented with cartoons in which the girl was the main character. The cartoons were presented one at a time, in the following order: $1,3,4,5,2,6,10,8,11,14,12,13,9,7$ (numbers refer to items in the Appendix). For each cartoon, the participant had to answer the question "What is the boy (girl) thinking?" The transcription of the answer was scored by two independent raters as 0 (wrong level of recursive thinking), 1 (right level of recursive thinking, but not entirely accurate, e.g. "the boy is thinking about the boy/him" instead of "himself", using a pronoun with unclear reference) or 2 (correct). Agreement between the two raters was good. Cohen's 
Kappa ranged from .66 to .93 (median $=.82$ ) across the 14 items at Time 1 and from .51 to $.90($ median $=.82)$ at Time 2 . For the present study, scores of 1 and 2 were collapsed (corresponding to the binary scoring system used by Miller et al., 1970). Scores on the recursive thinking test were available for all 299 participants at Time 1 and for 221 of the 222 participants at Time 2 . The test could not be administered to one participant, because she was unable to come to the lab.

HPA-axis activity. For the assessment of cortisol (nmol/l), seven saliva samples were collected by passively drooling into plastic vials (IBL-SaliCap®, Germany) directly or through a straw. Figure 1 depicts the timing of the samples and the mean cortisol concentrations in each sample at Time 1 and Time 2. Sample 1 was taken after the nature video (i.e. baseline sample). After the speech, six samples were taken to account for the fact that individuals differ in the timing of the cortisol response to a stressful event (Gunnar \& Talge, 2007). Sample 2 was collected directly after the speech and sample 3 was taken 10 minutes later. Samples 4 to 7 were collected at intervals of 5 minutes, so that the seventh and last saliva sample was taken at the end of the recovery period. Samples from one participant at one time of measurement were batched together for analysis.

The determination of cortisol in saliva was performed with a competitive electrochemiluminescence immunoassay ECLIA using a Modular Analytics E170 immunoassay analyzer from Roche Diagnostics (Mannheim, Germany). The sample volume was at least $20 \mu \mathrm{l}$. Missing values due to insufficient volume ranged between 0.5 and $13.4 \%$ of the samples $(M=4.6 \%)$. Missing values in samples 2 to 6 were interpolated by averaging the previous and the next sample. After interpolation, the percentage of missing values ranged from 0 to $5.3 \%(M=1.6 \%)$. In the statistical analyses, the natural logarithm of the cortisol concentrations was used, because the data were highly skewed. Outliers of more than 3 SD were winsorized. One multivariate outlier at Time 2 was deleted. 
Treatment of factors potentially influencing cortisol measures. At the beginning of the public speaking session, participants filled out a questionnaire on factors potentially influencing the cortisol concentration, including current medication usage, eating and drinking less than one hour before the start of the session and current phase of the menstrual cycle and the use of oral contraceptives in girls. Long-term use of medication was assessed with a health and medication history questionnaire, which participants had filled out at home. The use of any medication (regularly or accidentally on the day of the study), eating and/or drinking milk less than one hour before the public speaking session and use of oral contraceptives were statistically controlled for. We did not control for phase of the menstrual cycle. In adult studies, this variable is often used to control for fluctuations in estradiol, which affect the cortisol response. However, the phase of the menstrual cycle can only be determined for girls with a regular cycle, while the fluctuations in estradiol begin years before menarche (Shirtcliff, Dahl, \& Pollak, 2009). As the phase of the menstrual cycle could not be determined for the majority of girls in the present study (73\% at Time 1 and 53\% at Time 2), it did not seem useful as a control variable.

Pubertal status. Participants filled out a widely used self-report questionnaire: the Pubertal Development Scale (PDS; Petersen, Crockett, Richards, \& Boxer, 1988). Three items of the PDS were used to compute a pubertal development score (see Van den Bos et al., 2014). For girls, the items concerned menarche, pubic hair development and breast development. For boys, the items concerned voice change, pubic hair development and facial hair development. The individual items were scored on a scale from 1 to 4 , except for the item concerning menarche. This item was scored as 1 if the girl had not experienced menarche yet and as 4 if she had (Petersen et al., 1988). The overall pubertal development score was calculated by averaging the ratings on the three items. At Time 1, the PDS was completed by 288 participants $(97.6 \%)$ and the mean score was $2.1(S D=1.0)$ for boys and $2.7(S D=1.1)$ 
for girls. Cronbach's alpha was .86 for boys and .85 for girls. At Time 2, the PDS was completed by 216 participants $(99.5 \%)$ and the mean score was $2.7(S D=1.0)$ for boys and $3.3(S D=0.9)$ for girls. Cronbach's alpha was .89 for boys and .82 for girls.

Raven's Standard Progressive Matrices. Raven's SPM is a measure of Spearman's $\mathrm{g}$ (Raven, 2009). It was included as a control variable to test whether relations with recursive thinking are independent of relations with age-related improvements in general cognitive functioning. As noted by Diamond (2013) scores on the SPM are highly correlated with measures of executive functions. The test comprises 60 items. Each item consists of a large figure, from which one piece is missing, and several alternatives for the missing piece. The participant has to choose the one piece that correctly completes the figure, because it follows the pattern presented in the figure (Raven, 2009). The items are divided into five sets of 12 . In each set, a different principle is used to create the patterns. The number of alternatives also varies between the sets: there are 6 alternatives in sets $A$ and $B$ and 8 alternatives in sets $C, D$ and E. Within each set, the items are presented in increasing order of difficulty (Raven, 2009).

The SPM was administered in Wave 2 of the SAND-study (one year after Time 1 and one year before Time 2), during a single session in which participants also filled out questionnaires. Elementary school participants were tested in the classroom at school. Secondary school participants were tested in lecture rooms at the university. The SPM was presented in a booklet. Participants recorded their answers on a response form. The score on the SPM was computed by summing the number of correct pieces chosen. Raw scores were used, because they reflect the improvement of executive functions with age. Scores were available for 217 of the 221 participants in the present sample.

\section{Statistical Analyses}

Trajectories of recursive thinking. Our hypothesis concerned a group of participants who shifted from non-recursive thinking to recursive thinking in the two-year interval of our 
study. We used latent class analysis (LCA) to establish whether or not our sample contained such a group. With LCA, subgroups of participants are detected in the data based on similar response patterns in the recursive thinking task. The number of subgroups is determined by comparing how well different solutions fit the data. Although the approach does not guarantee detection of a response pattern that reflects the transition to recursive thinking, it has the advantage of being more objective than setting criteria for assigning participants to subgroups by hand.

The flexmix package (Leisch, 2004) was used to run the LCA in R 2.5.1 (R development core team, 2007). The analysis was done on individuals binomial (correct, incorrect) sum scores on each of the three types of item in the recursive thinking test at Time 1 and Time 2 (i.e. number of correct answers for action items at Time1, one-loop items at Time 1, two-loop items at Time 1, action items at Time 2, one-loop items at Time 2 and twoloop items at Time 2). Our application of LCA included three steps. First, we repeatedly fitted models with one up to ten subgroups with random start-values. Second, the best converging solutions of these models were compared by means of Bayesian Information Criterion (BIC) and the best solution, with the lowest BIC, was selected. Third, we calculated the posterior probabilities to assign each participant to his or her most likely subgroup.

Including both time-points in the LCA allowed to detect subgroups in our data that maintained a similar pattern of recursive thinking across Time 1 and Time 2, and subgroups that changed response patterns of recursive thinking across time. As noted above, we were interested in participants whose response pattern indicated a transition from non-recursive thinking at Time 1 to recursive thinking at Time 2 . In the main analyses, participants assigned to this trajectory were compared with participants not assigned to this trajectory (i.e. participants assigned to any other trajectory). This was coded as a dummy variable Transition $(0=$ no, $1=$ yes $)$ 
Main analysis. We aimed to investigate whether the magnitude and timing of the cortisol response were related to the transition to recursive thinking. To address these questions, we compared participants who experienced the transition from non-recursive thinking at Time 1 to recursive thinking at Time 2 with participants who did not experience this transition from Time 1 to Time 2 . A change in the cortisol response from Time 1 to Time 2 for participants who transitioned to recursive thinking, but not for other participants (i.e. a Transition $\mathrm{x}$ Time interaction) provides evidence that the cortisol response is related to the transition to recursive thinking.

We analyzed our data using regression analysis with clustered bootstrap (Cameron, Gelbach, \& Miller, 2008; De Rooij, 2013; Harden, 2011), because this technique is suitable for time-varying predictors (De Rooij, 2013). It allows for controlling the cortisol response at Time 1 for confounding factors at Time 1 and the cortisol response at Time 2 for confounding factors at Time 2. Regression analysis was done on the data from Time 1 and Time 2 combined. The clustered bootstrap procedure (De Rooij, 2013; Sherman \& Le Cessie, 1997) was used for statistical inference. Intercepts and regression weights were estimated as in standard regression analysis, but the standard errors were derived by bootstrapping. From the total data set, 10,000 bootstrap samples of the same size as the original set were drawn randomly with replacement. To deal with the dependency between measurements of the same individual, the bootstrap was clustered: individuals were sampled rather than cases, so that, if the individual was assessed at both times, both measurements were included in the sample (De Rooij, 2013).

The regression model included the following explanatory variables: gender $(1=$ male, $0=$ female $)$, medication $(1=$ yes, $0=$ no $)$, oral contraceptives $(1=$ yes, $0=$ no $)$, recent food intake ( $1=$ yes, $0=$ no), sum score on the SPM, mean score on the PDS, age at Time 1 (T1 Age, centered at the age of the youngest participant), time since first assessment (Time: 
current age minus age at Time 1), the transition to recursive thinking from Time 1 to Time 2 (Transition: $1=$ yes, $0=$ no) and, crucially, the Time $\mathrm{x}$ Transition interaction. Because the purpose of this analysis was to rigorously control the effect of recursive thinking for other developments, T1 Age, mean score on the PDS and sum score on the SPM were included in the same model. However, variance inflation factors of 3.7 for T1 Age and 3.8 for PDS indicated that the unique contributions of these variables should be interpreted with caution.

Cortisol response indices. The dependent variables were three indices of the cortisol response to the LPST: the Area Under the Curve with respect to increase (AUCi; Pruessner, Kirschbaum, Meinlschmid, \& Hellhammer, 2003), the speech anticipation response and the speech delivery response. AUCi is an index of the overall stress response, which is sensitive to both its height and its duration. It represents the increase in concentration relative to a baseline. Because participants knew beforehand that they would have to give a speech, the pre-task concentration was not a valid baseline. The concentration at recovery, which is the best indication of a participant's resting level, was used instead (see Westenberg et al., 2009). Speech anticipation and speech delivery represent the cortisol responses to two phases of the task. The speech anticipation response was defined as the pre-task concentration minus the concentration at recovery. The speech delivery response was defined as the maximum concentration in post-task samples minus the pre-task concentration.

\section{Results}

\section{Trajectories of Recursive thinking}

To identify trajectories of recursive thinking over time, we attempted to fit models containing 1 to 10 classes to the data from both Time 1 and Time 2 for 221 participants. The model with 5 classes had the best fit according to the Bayesian Information Criterion (BIC = 3277.031). Table 2 shows the estimated probability of a correct answer on each type of item 
at Time 1 and Time 2, the total number of participants, number of males, mean age at Time 1 , mean PDS-score at Time 1 and Time 2 and mean SPM score.

The classes can be interpreted as trajectories of recursive thinking. Participants in the first trajectory changed from action level to one-loop level. At Time 1, they were more often correct than not on action items, but not on one-loop recursion or two-loop recursion items.

At Time 2, they performed almost perfectly on action items and were more often correct than not on one-loop recursion items, but performed still poorly on two-loop recursion items (see Table 2). Participants in the second trajectory remained at one-loop level and showed no improvement from Time 1 to Time 2. At both times, they performed near ceiling on action items and were more often correct than not on one-loop recursion items, but not on two-loop recursion items (see Table 2). Participants in the third trajectory changed from one-loop level to two-loop level. At Time 1, they were more often correct than not on action items and oneloop recursion items, but not on two-loop recursion items. At Time 2, they performed almost perfectly on action items and one-loop recursion items and were more often correct than not on two-loop recursion items (see Table 2). Performance in the fourth and fifth trajectory indicated two-loop recursive thinking level. Participants in both trajectories performed near ceiling on action items and one-loop recursion items and were more often correct than not on two-loop recursion items at Time 1 . However, participants in the fourth trajectory showed no further improvement from Time 1 to Time 2, whereas participants in the fifth trajectory improved to as good as perfect performance on all items at Time 2 (see Table 2).

\section{Effects of recursive thinking on the cortisol response indices}

In our main analyses, participants who transitioned from non-recursive thinking at Time 1 to recursive thinking at Time 2 (i.e. transition; action to one-loop trajectory) were compared with participants who were at recursive thinking at both times (i.e. no transition; all other trajectories). We investigated whether the transition to recursive thinking from Time 1 
to Time 2 was related to changes in the magnitude and timing of the cortisol response from Time 1 to Time 2 (i.e. a Time $\mathrm{x}$ Transition interaction). Table 3 shows the regression weights of the explanatory variables for each dependent variable.

Magnitude of the response. For the overall cortisol response, AUCi, the Time $\mathrm{x}$ Transition interaction was not significant, indicating that the magnitude of the overall cortisol response was not related to the transition to recursive thinking. Only the control variables age at Time 1 and recent food intake were significant. The overall cortisol response was larger in older participants and smaller in participants who had consumed food or dairy products in the hour before the lab session.

Timing of the response. For the cortisol response to speech anticipation, the regression analysis with clustered bootstrap showed a significant interaction between Time and Transition (see Figure 2). Participants who transitioned from non-recursive thinking at Time 1 to recursive thinking at Time 2 showed an increase of the speech anticipation response from Time 1 to Time 2, whereas other participants did not. The control variables age at Time 1 and recent food intake were also significant. The cortisol response to speech anticipation was larger for older participants and smaller for participants who had consumed food or diary products in the hour before the lab session. The speech anticipation response was marginally significantly smaller for boys than for girls.

For the cortisol response to speech delivery, the regression analysis with clustered bootstrap showed a marginally significant interaction between Time and Transition (see Figure 3). Participants who transitioned from non-recursive thinking at Time 1 to recursive thinking at Time 2 showed a small decrease of the speech delivery response from Time 1 to Time 2, whereas other participants showed an increase. The control variable recent food intake was significant: participants who had consumed food or dairy products in the hour before the lab session showed a larger cortisol response to speech delivery. Gender and score 
on the SPM were marginally significant: the speech delivery response was somewhat larger in boys than in girls and somewhat lower in participants with higher scores on the Standard Progressive Matrices.

The pattern of an increasing speech anticipation response and a decreasing speech delivery response in participants who transitioned from non-recursive thinking at Time 1 to recursive thinking at Time 2 is in line with the possibility that the transition to recursive thinking affects the timing of the cortisol response. Both findings could be due to an increase in cortisol concentrations before the speech. However, alternative explanations are possible, because speech anticipation and speech delivery were both computed by taking the difference in cortisol concentration between two saliva samples. For example, the increasing speech anticipation response may also be due to a decrease in cortisol concentrations at recovery. A final regression analysis with clustered bootstrap was done to compare the Time $\mathrm{x}$ Transition effects on the pre-task sample, the peak sample and the recovery sample. Sample was coded using two dummy variables: pretask $(1=$ yes, $0=$ no $)$ and peak $(1=$ yes, $0=$ no $)$, with recovery being the reference category. The regression model included the explanatory variables used in the previous analyses as well as pretask, peak, their two-way interactions with Time, their two-way interactions with Transition and their three-way interactions with Time and Transition.

The results, summarized in Table 3, showed a significant Pretask x Time x Transition interaction. As illustrated by Figure 4, the interaction indicated that, for participants who transitioned to recursive thinking from Time 1 to Time 2 (black lines), the increase in cortisol concentration from Time 1 (dotted lines) to Time 2 (solid lines) was larger for the pretask sample than for the recovery sample. This finding corroborates that the transition to recursive thinking is associated with an increase in cortisol concentrations before the task. 
The analysis also showed that cortisol concentrations in the pre-task sample and the peak sample were significantly higher than in the recovery sample (averaged over both times and all participants). Cortisol concentrations (averaged over all samples) were significantly higher for participants at higher levels of pubertal development. In addition, average concentrations were marginally higher for boys than for girls. Peak concentrations (averaged over both times) were marginally significantly lower in participants who transitioned to recursive thinking than in others.

\section{Discussion}

Previous studies have shown that the magnitude of the cortisol response to socialevaluative stressors increases with age and pubertal development during adolescence (Gunnar, Wewerka et al., 2009; Stroud et al., 2009; Sumter et al., 2010; Van den Bos et al., 2014). The present study is the first to relate the adolescent development of the cortisol response to another aspect of normative development: social cognition. The results showed that sociocognitive development was a significant predictor of the anticipatory cortisol response, which increased from Time 1 to Time 2 for adolescents who made the transition from non-recursive thinking to recursive thinking during this two-year period. The increase associated with this transition was independent from the effects of age and two other developments occurring in adolescence: puberty and improvements in general cognitive functioning. The cortisol response to speech delivery showed a slight decrease associated with the transition to recursive thinking, while the overall cortisol response was not affected. This pattern of results indicates that recursive thinking affects the timing of the cortisol response rather than its magnitude. Participants at non-recursive levels of thinking showed relatively low cortisol concentrations before the task and an increase during the task, whereas participants at recursive levels of thinking showed relatively high cortisol concentrations before the task and less of an increase during the task. 
A specific relation between recursive thinking and anticipation of social evaluation may explain two other findings in the literature. In the study by Gunther Moor et al. (2010), activation of the $\mathrm{mPFC}$ and the striatum were positively related with age while participants were waiting for acceptance feedback and expected to be liked, but not in response to the actual feedback. In the study by Stroud et al. (2009), adolescents showed heightened cortisol levels before a social interaction task, although they did not show a cortisol response to actually performing the task. These findings suggest that, with the development of recursive thinking, adolescents come to perceive social-evaluative threat prior to entering a situation. Anticipatory responses are elicited, which may sufficiently prepare them for a potential threat, so that no further responses are needed when they face the situation.

Our finding that the cortisol response in anticipation of a social-evaluative situation increased with the transition to recursive thinking may also help to explain why social anxiety disorder, which is characterized by excessive fear of social evaluation, tends to have its onset in adolescence (American Psychiatric Association, 2013). Several authors have suggested that socio-cognitive development may increase the risk for social anxiety in vulnerable individuals (Bokhorst et al., 2008; Gren-Landell et al., 2009; Ollendick \& Hirshfeld-Becker, 2002). The present study suggests that the emergence of recursive thinking leads to earlier recognition of social-evaluative threat. Earlier recognition, in turn, may offer these adolescents more opportunity to avoid social situations, which is thought to exacerbate their anxiety (Rapee \& Spence, 2004) and interfere with daily life, increasing their need for treatment (Miers, Blöte, Heyne, \& Westenberg, 2014). Future research may investigate this potential mechanism in children at risk.

As noted above, the transition to recursive thinking did not increase the cortisol response to the actual public speaking task. This finding raises the question whether children at non-recursive levels of thinking understand that they are subject to social evaluation once 
they have entered the situation. Even children of five or six years old show a cortisol response to a social-evaluative stressor (De Weerth, Zijlmans, Mack, \& Breijers, 2013). This may be because the situation itself provides cues that make them realize the social-evaluative threat, such as being watched by others. However, being the center of attention may also be somewhat stressful in itself. Lewis (2005) noted that embarrassment may result either from negative evaluation or from mere exposure to the attention of others. Another cue, in the context of public speaking, is that participants are told that their performance will be evaluated. On the one hand, this may prime participants to realize the potential social consequences of their performance. On the other hand, the explicit threat of performance evaluation may in itself be sufficient to elicit a cortisol response. Public speaking has indeed been characterized as both a social-evaluative stressor and a performance stressor (Dickerson \& Kemeny, 2004; Stroud et al., 2009). More research varying the characteristics of socialevaluative situations is needed to clarify whether children at non-recursive levels of thinking become aware of acute social-evaluative threat.

The findings that the magnitude of the overall cortisol response increases with pubertal development (Van den Bos et al., 2014), but is not affected by the transition to recursive thinking are in line with suggestions in the neurodevelopmental literature that the increasing sensitivity to social evaluation in adolescence is mainly due to sensitization of the affective node by pubertal hormones (Burnett et al., 2011; Haller et al., 2014; Nelson et al., 2005; Somerville, 2013). The increase in anticipation of social evaluation with the transition to recursive thinking may be related to developmental changes in the cognitive-regulatory node, which Nelson et al. (2005) related to theory of mind operations and executive functions. The recursive thinking test (Miller et al., 1970) was designed to measure an aspect of theory of mind but - like any other theory of mind test - also makes demands on executive functions. In the present study, the score on the Raven SPM was included to control for the contribution of 
general cognitive functioning. Hence, the increase in the anticipatory cortisol response can be ascribed to changes in theory of mind operations; earlier realization of social-evaluative threat is related to emergence of the ability to think about other people's thoughts.

Some authors have suggested that sensitivity to social evaluation decreases with further maturation of the cognitive-regulatory node in late adolescence and young adulthood (Burnett et al., 2011; Nelson et al., 2005). The present study found a marginally significant negative relation between the sum score on the SPM, which is highly correlated with executive function (Diamond, 2013), and the cortisol response to speech delivery. This might suggest that the actual task of giving a speech becomes more manageable and less stressful with improvement of executive functions. Future studies focusing on late adolescence and young adulthood may find stronger support for a negative relation between executive functions and the cortisol response to social evaluation. Moreover it would be interesting to use specific measures of inhibition, working memory and cognitive flexibility instead of a global index of executive functions such as the SPM. Working memory in particular may be a good candidate, because its development continues into young adulthood (Huizinga et al., 2006).

With regard to the development of recursive thinking, the present study provided additional evidence for the claim by Miller et al. (1970) that a series of consecutive steps can be distinguished. Latent class analysis produced groups that were easily interpretable as trajectories of recursive thinking. Over a two-year interval, participants either remained at the same level of recursive thinking or progressed to the next level. Of those participants who had grasped two-loop recursive thinking at Time 1, nearly one-third moved on to near-perfect performance. In the other participants development may still be ongoing: the total score on the recursive thinking test was found to increase linearly throughout adolescence (Van den Bos et al., 2016). Alternatively, there may be lasting individual differences in proficiency at 
recursive thinking. Further research examining individual differences in recursive thinking in (young) adults is needed to settle this issue.

In the present study, only post-task recovery concentrations were available as indices of participants' resting levels of cortisol. Pre-task concentrations are elevated in the LPST, because participants are informed a week in advance that they will have to give a speech that will be recorded and evaluated by age-peers. Following this protocol in both waves of our longitudinal study was preferable to using an impromptu speech task, which would be a surprise the first time, but may be anticipated the next. In everyday life, public speaking is usually anticipated. The present results demonstrate the importance of studying responses to both speech anticipation and speech delivery.

A limitation of the present study is that our sample proved to be relatively 'old' for studying the transition from non-recursive thinking to recursive thinking: most participants turned out to be already at recursive levels of thinking at Time 1 . The findings would be strengthened by replication in a younger sample. Another limitation is the use of a self-report measure of pubertal development, which is less reliable than assessment by a trained physician. Shirtcliff et al. (2009) showed that PDS scores correlated moderately with a physical exam. Boys and girls who matured relatively early (or late) compared with their agepeers tended to over-report (or underreport) their pubertal status. However, the PDS and the physical exam were similarly related to levels of pubertal hormones. Moreover, we controlled for age and improvements in general cognitive functioning in addition to pubertal development. This strengthens the evidence that part of the increase in the anticipatory cortisol response to social evaluation is specifically related to the transition to recursive thinking.

The longitudinal design of the study lends credence to our findings, by demonstrating a relation between a change in recursive thinking and a change in the timing of the cortisol 
response to social evaluative-threat within the same individuals. However, the design was correlational and the changes were assessed simultaneously. Therefore, the present study did not provide evidence for a causal relation. Demonstrating causality in future research will be challenging. Future studies might investigate whether training in recursive thinking leads to an increase in the cortisol response to social evaluation. However, it is at present unknown whether a transition from non-recursive thinking to recursive thinking can be brought about by training. 


\section{References}

American Psychiatric Association (2013). Diagnostic and Statistical Manual for Mental Disorders, 5th edition (DSM-5). Arlington, VA: American Psychiatric Publishing.

Blakemore, S. J., \& Mills, K. L. (2014). Is adolescence a sensitive period for sociocultural processing? Annual Reviews of Psychology, 65, 187-207. DOI: 10.1146/annurevpsych-010213-115202

Blöte, A. W., Bokhorst, C. L., Miers, A. C., \& Westenberg, P. M. (2012). Why are socially anxious adolescents rejected by peers? The role of subject-group similarity characteristics. Journal of Research on Adolescence, 22, 123-134. DOI: 10.1111/j.1532-7795.2011.00768.x

Bokhorst, C.L., Westenberg, P. M., Oosterlaan, J., \& Heyne, D. A. (2008). Changes in social fears across childhood and adolescence: Age-related differences in the factor structure of the Fear Survey Schedule for Children-Revised. Journal of Anxiety Disorders, 22, 135-142. DOI: $10.1016 /$ j.janxdis.2007.01.014

Burnett, S., Sebastian, C., Cohen Kadosh, K., \& Blakemore, S. J. (2011). The social brain in adolescence: Evidence from functional magnetic resonance imaging and behavioural studies. Neuroscience and Biobehavioral Reviews, 35, 1654-1664.

DOI:10.1016/j.neubiorev.2010.10.011

Cameron, A. C., Gelbach, J. B., \& Miller, D. L. (2008). Bootstrap based improvements for inference with clustered errors. Review of Economics and Statistics, 90, 414-427. DOI:10.1162/rest.90.3.414

Choudhury, S., Blakemore, S. J., \& Charman, T. (2006). Social cognitive development during adolescence. SCAN, 1, 165-174. DOI: 10.1093/scan/ns1024

De Rooij, M. (2013). Standard regression models for repeated measurement and longitudinal data. Unpublished manuscript. 
De Weerth, C., Zijlmans, M. A. C., Mack, S., \& Beijers, R. (2013). Cortisol reactions to a social evaluative paradigm in 5- and 6-year-old children. Stress, 16, 65-72. DOI:10.3109/10253890.2012.684112

Diamond, A. (2013). Executive functions. Annual Reviews of Psychology, 64, 135-168. DOI: 10.1146/annurev-psych-113011-143750

Dickerson, S. S., \& Kemeney, M. E. (2004). Acute stressors and cortisol responses: A theoretical integration and synthesis of laboratory research. Psychological Bulletin, 130, 355-391. DOI: 10.1037/0033-2909.130.3.355

Dumontheil, I., Apperly, I. A., \& Blakemore, S.J. (2010). Online usage of theory of mind continues to develop in late adolescence. Developmental Science, 13, 331-338. DOI: 10.1111/j.1467-7687.2009.00888.x

Eliot, J., Lovell, K., Dayton, C. M., \& McGrady, B. F. (1979). A further investigation of children's understanding of recursive thinking. Journal of Experimental Child Psychology, 28, 149-157. DOI:10.1016/0022-0965(79)90108-5

Gren-Landell, M., Tillfors, M., Furmark, T., Bohlin, G., Andersson, G., \& Svedin, C. G. (2009). Social Phobia in Swedish Adolescents. Social psychiatry and psychiatric epidemiology, 44, 1-7. DOI 10.1007/s00127-008-0400-7

Gunnar, M. R. \& Talge, N. M. (2007). Neuroendocrine measures in developmental research. In: Schmidt, L. A., Segalowitz, S. J. (Eds.), Developmental Psychophysiology. Cambridge University Press, Cambridge, UK, pp. 343-366.

Gunnar, M. R., Talge, N. M., \& Herrera, A. (2009). Stressor paradigms in developmental studies: what does and does not work to produce mean increases in salivary cortisol. Psychoneuroendocrinology, 34, 953-967. DOI: 10.1016/j.psyneuen.2009.02.010

Gunnar, M. R., Wewerka, S., Frenn, K., Long, J. D., \& Griggs, C. (2009). Developmental changes in hypothalamus-pituitary-adrenal activity over the transition to adolescence: 
Normative changes and associations with puberty. Development and Psychopathology, 21, 69-85. DOI: 10.1017/S0954579409000054

Gunther Moor, B., Bos, M. G. N., Crone, E. A., \& Van der Molen, M. W. (2014). Peer rejection cues induce cardiac slowing after transition into adolescence. Developmental Psychology, 50, 947-955. DOI: 10.1037/a0033842

Gunther Moor, B., Van Leijenhorst, L., Rombouts, S. A. R. B., Crone E. A., \& Van der Molen, M. W. (2010). Do you like me? Neural correlates of social evaluation and developmental trajectories. Social Neuroscience, 5, 461-482.

DOI:10.1080/17470910903526155

Haller, S. P. W., Cohen Kadosh, K., \& Lau, J. Y. F. (2014). A developmental angle to understanding the mechanisms of biased cognitions in social anxiety. Frontiers in Human Neuroscience, 7. DOI:10.3389/fnhum.2013.00846

Harden, J. (2011). A bootstrap method for conducting statistical inference with clustered data. State Politics \& Policy Quarterly, 11, 223-246. DOI: 10.1177/1532440011406233

Hollenstein, T., McNeely, A., Eastabrook, J., Mackey, A., \& Flynn, J. (2012). Sympathetic and parasympathetic responses to social stress across adolescence. Developmental Psychobiology, 54, 207-214. DOI:10.1002/dev.20582

Huizinga, M., Dolan, C. V., \& Van der Molen, M. W. (2006). Age-related change in executive function: Developmental trends and a latent variable analysis. Neuropsychologia, 44, 2017-2036. DOI: 10.1016/j.neuropsychologia.2006.01.010

Klimes-Dougan, B., Hastings, P. D., Granger, D. A., Usher, B. A., \& Zahn-Waxler, C. (2001). Adrenocortical activity in at-risk and normally developing adolescents: Individual differences in salivary cortisol basal levels, diurnal variation, and responses to social challenges. Development and Psychopathology, 13, 695-719. DOI: $10.1017 /$ S0954579401003157 
Landry, M. O., \& Lyons-Ruth, K. (1980). Recursive structure in cognitive perspective taking. Child Development, 51, 386-394. DOI: 10.2307/1129271

Leisch, F. (2004). FlexMix: A general framework for finite mixture models and latent class regression in R. Journal of Statistical Software, 11(8). http://jstatsoft.org/v11/i08/

Lewis, M. (2005). Origins of the self-conscious child. In W. R. Crozier, \& L. E. Alden (Eds.), The essential handbook of social anxiety for clinicians (pp. 81-98). Chichester, England: John Wiley \& Sons Ltd.

Miers, A. C., Blöte, A. W., De Rooij, M., Bokhorst, C. L., \& Westenberg, P. M. (2013). Trajectories of social anxiety during adolescence and relations with cognition, social competence, and temperament. Journal of Abnormal Child Psychology, 41, 97-110. DOI:10.1007/S10802-012-9651-6

Miers, A. C., Blöte, A. W., Heyne, D. A., \& Westenberg, P. M. (2014). Developmental pathways of social avoidance across adolescence: The role of social anxiety and negative cognition. Journal of Anxiety Disorders, 28, 787-794.

DOI:10.1016/j.janxdis.2014.09.008

Miller, P. H., Kessel, F. S., \& Flavell, J. H. (1970). Thinking about people thinking about people thinking about...: A study of social cognitive development. Child Development, 41, 613-623. DOI:10.1111/j.1467-8624.1970.tb01018.x

Müeller, U. \& Overton, W. F. (2010). Thinking about thinking - thinking about measurement: A Rasch analysis of recursive thinking. Journal of Applied Measurement, 11, 78-90.

Nelson, E. E., Leibenluft, E., McClure, E. B., \& Pine, D. S. (2005). The social re-orientation of adolescence: A neuroscience perspective on the process and its relation to psychopathology. Psychological Medicine, 35, 163-174.

DOI:10.1017/S0033291704003915 
Ollendick, T. H., \& Hirshfeld-Becker, D. R. (2002). The developmental psychopathology of social anxiety disorder. Biological Psychiatry, 51, 44-58. DOI:10.1016/S00063223(01)01305-1

Oppenheimer, L. (1986). Development of recursive thinking: Procedural variations. International Journal of Behavioral Development, 9, 401-411. DOI:10.1177/016502548600900309

Petersen, A. C., Crockett, L., Richards, M., \& Boxer, A. (1988). A self-report measure of pubertal status: Reliability, validity and initial norms. Journal of Youth and Adolescence, 17, 117-133. DOI:10.1007/BF01537962

Pruessner, J. C., Kirschbaum, C., Meinlschmid, G., \& Hellhammer, D.H. (2003). Two formulas for computation of the area under the curve represent measures of total hormone concentration versus time-dependent change. Psychoneuroendocrinology, 28, 916-931. DOI:10.1016/S0306-4530(02)00108-7

R development core team (2007). R: A language and environment for statistical computing. R foundation for statistical computing: Vienna, Austria.

Rapee, R. M., \& Spence, S. H. (2004). The etiology of social phobia: Empirical evidence and an initial model. Clinical Psychology Review, 24, 737-767.

DOI:10.1016/j.cpr.2004.06.004

Raven, J. (2009). The Raven Progressive Matrices and measuring aptitude constructs. The International Journal of Educational and Psychological Assessment, 2, 2-38.

Sherman, M. \& Le Cessie, S. (1997). A comparison between bootstrap methods and generalized estimating equations for correlated outcomes in generalized linear models. Communications in Statistics - Simulation and Computation, 26, 901-925. DOI:10.1080/03610919708813417 
Shirtcliff, E. A., Dahl, R. E., \& Pollak, S. D. (2009). Pubertal development: correspondence between hormonal and physical development. Child Development, 80, 327-337. DOI: 10.1111/j.1467-8624.2009.01263.x

Silk, J. S., Stroud, L. R., Siegle, G. J., Dahl, R. E., Lee, K. H., \& Nelson, E. E. (2012). Peer acceptance and rejection through the eyes of youth: pupillary, eyetracking and ecological data from the Chatroom Interact task. SCAN, 7, 93-105. DOI:10.1093/scan/nsr044

Somerville, L. H. (2013). The teenage brain: Sensitivity to social evaluation. Current Directions in Psychological Science, 22, 121-127. DOI:10.1177/0963721413476512

Somerville, L. H., Jones, R. M., Ruberry, E. J., Dyke, J. P., Glover, G., \& Casey, B. J. (2014). The medial prefrontal cortex and the emergence of self-conscious emotion in adolescence. Psychological Science, 24, 1554-1562. DOI:10.1177/0956797613475633

Stroud, L. R., Foster, E., Papandonatos, G. D., Handwerger, K., Granger, D.A., Kivlighan, K. T., \& Niaura, R. (2009). Stress response and the adolescent transition: Performance versus peer rejection stressors. Development and Psychopathology, 21, 47-68. DOI:10.1017/S0954579409000042

Sumter, S. R., Bokhorst, C. L., Miers, A. C., Van Pelt, J., \& Westenberg, P. M. (2010). Age and puberty differences in stress responses during a public speaking task: Do adolescents grow more sensitive to social evaluation? Psychoneuroendocrinology, 35, 1510-1516. DOI:10.1016/j.psyneuen.2010.05.004

Van den Bos, E., De Rooij, M., Miers, A. C., Bokhorst, C. L., \& Westenberg, P. M. (2014). Adolescents' increasing stress response to social evaluation: Pubertal effects on cortisol and alpha-amylase during public speaking. Child Development, 85, 220-236. DOI:10.1111/cdev.12118 
Van den Bos, E., De Rooij, M., Sumter, S. R., \& Westenberg, P. M. (2016). Continued development of recursive thinking in adolescence: Longitudinal analyses with a revised recursive thinking test. Cognitive Development, 37, 28-41.

DOI:10.1016/j.cogdev.2015.11.002

Vasey, M. W., Crnic, K. A., \& Carter, W. G. (1994). Worry in childhood. A developmental perspective. Cognitive Therapy and Research, 18, 529-549.

DOI:10.1007/BF02355667

Veith, D. L. (1980). Recursive thinking and the self-concepts of preadolescents. Journal of Genetic Psychology, 137, 233-246.

Vetter, N. C., Leipold, K., Kliegel, M., Phillips, L. H., \& Altgassen, M. (2013). Ongoing development of social cognition in adolescence. Child Neuropsychology, 19, 615-629. DOI: $10.1080 / 09297049.2012 .718324$

Weems, C. F., \& Costa, N. (2005). Developmental differences in the expression of childhood anxiety symptoms and fear. Journal of the American Academy of Child and Adolescent Psychiatry, 44, 656-663. DOI:10.1097/01.chi.0000162583.25829.4b

Westenberg, P. M., Bokhorst, C. L., Miers, A. C., Sumter, S. R., Kallen, V. L., Van Pelt, J., \& Blöte, A. W. (2009). A prepared speech in front of a pre-recorded audience: Subjective, physiological, and neuroendocrine responses to the Leiden Public Speaking Task. Biological Psychology, 82, 116-124.

DOI:10.1016/j.biopsycho.2009.06.005

Westenberg, P. M., Drewes, M. J., Goedhart, A. W., Siebelink, B. M., \& Treffers, Ph. D. A.. (2004). A developmental analysis of self reported fears in late childhood through mid adolescence: Social-evaluative fears on the rise? Journal of Child Psychology and Psychiatry, 45, 481-495. DOI:10.1111/j.1469-7610.2004.00239.x 
Westenberg, P. M., Gullone, E., Bokhorst, C. L., Heyne, D. A., \& King, N. J. (2007). Social evaluation fear in childhood and adolescence: Normative developmental course and continuity of individual differences. British Journal of Developmental Psychology, 25, 471-483. DOI:10.1348/026151006X173099

Westenberg, P. M., Siebelink, B. M., Warmenhoven, N. J. C., \& Treffers, Ph. D. A. (1999). Separation anxiety and overanxious disorders: Relations to age and level of psychosocial maturity. Journal of the American Academy of Child and Adolescent Psychiatry, 38(8), 1000-1007. DOI:10.1097/00004583-199908000-00016 
Table 1.

Distribution of Participants over Grade Levels in Primary and Secondary School at Time 1.

\begin{tabular}{lccl}
\hline Grade & n & n male & Mean age \\
& \multicolumn{3}{c}{ Primary school } \\
6 & 38 & 17 & $9.74(0.41)$ \\
7 & 44 & 29 & $10.91(0.43)$ \\
8 & 44 & 20 & $11.91(0.40)$ \\
& & Secondary school & \\
1 & 43 & 23 & $13.12(0.29)$ \\
2 & 42 & 23 & $14.10(0.51)$ \\
3 & 41 & 21 & $15.33(0.49)$ \\
4 & 47 & 21 & $16.48(0.48)$ \\
\hline Note. Standard deviations of age are in parenthesis.
\end{tabular}


Table 2.

Response Pattern and Participant Characteristics for Each Trajectory of Recursive Thinking from Time 1 to Time 2.

\begin{tabular}{|c|c|c|c|c|c|c|c|c|c|c|c|c|}
\hline \multirow[t]{3}{*}{ Trajectory } & \multicolumn{3}{|c|}{ Time 1} & \multicolumn{3}{|c|}{ Time 2} & \multirow[b]{2}{*}{$\mathrm{n}$} & \multirow[b]{2}{*}{$\mathrm{n}$} & \multirow[b]{2}{*}{$\mathrm{T} 1$} & \multirow[b]{2}{*}{$\mathrm{T} 1$} & \multirow[b]{2}{*}{$\mathrm{T} 2$} & \multirow{3}{*}{ SPM } \\
\hline & $\mathrm{p}($ action $)$ & $\mathrm{p}$ (one- & p(two- & $\mathrm{p}$ (action) & $\mathrm{p}$ (one- & p(two- & & & & & & \\
\hline & & loop) & loop) & & loop) & loop) & total & male & Age & PDS & PDS & \\
\hline Action to one- & .718 & 0.465 & 0.096 & 0.970 & 0.642 & 0.156 & 34 & 17 & 12.2 & 2.06 & 2.65 & 44.7 \\
\hline loop & & & & & & & & & (2.3) & (1.1) & $(1.0)$ & (6.7) \\
\hline One-loop: no & .977 & 0.849 & 0.254 & 0.940 & 0.763 & 0.232 & 61 & 24 & 12.9 & 2.41 & 2.91 & 46.6 \\
\hline improvement & & & & & & & & & $(2.4)$ & $(1.2)$ & (1.1) & $(5.5)$ \\
\hline One-loop to & 0.764 & 0.632 & 0.192 & 0.975 & 0.983 & 0.838 & 25 & 19 & 12.6 & 2.15 & 2.88 & 46.7 \\
\hline two-loop & & & & & & & & & $(2.1)$ & $(0.9)$ & $(1.0)$ & $(5.8)$ \\
\hline Two-loop: no & 0.931 & 0.915 & 0.615 & 0.943 & 0.920 & 0.647 & 69 & 33 & 13.5 & 2.61 & 3.06 & 48.7 \\
\hline improvement & & & & & & & & & (2.4) & (1.1) & $(1.0)$ & (6.9) \\
\hline Two-loop to & 0.994 & 0.968 & 0.793 & 1.000 & 0.999 & 1.000 & 32 & 21 & 14.4 & 2.74 & 3.47 & 51.6 \\
\hline perfect & & & & & & & & & (1.5) & (1.0) & $(0.7)$ & (5.4) \\
\hline
\end{tabular}

Note. Standard deviations are in parenthesis. $\mathrm{p}=$ estimated probability of a correct response, $\mathrm{T} 1=$ Time 1 , T2 = Time 2, PDS = mean score on the pubertal development scale (Petersen et al., 1988), SPM is sum score on the Raven Standard Progressive Matrices. 
Table 3.

Regression Weights of the Explanatory Variables for Three Difference Scores and Absolute Cortisol Concentrations.

Difference scores

Absolute

\begin{tabular}{|c|c|c|c|c|}
\hline & & & & \\
\hline Explanatory variables & AUCi & Speech anticipation & Speech delivery & concentration \\
\hline Male & -1.53 & $-0.09 \dagger$ & $0.08 \dagger$ & $0.11 \dagger$ \\
\hline Medication & -0.74 & -0.03 & 0.07 & 0.07 \\
\hline Oral contraceptive & -2.96 & -0.05 & -0.09 & 0.16 \\
\hline Recent food intake & $-6.62 * *$ & $-0.32 * * *$ & $0.12 *$ & -0.07 \\
\hline SPM & -0.03 & 0.00 & $-0.01 \dagger$ & 0.00 \\
\hline PDS & 0.47 & 0.03 & 0.02 & $0.13 * *$ \\
\hline T1 Age & $1.74 * *$ & $0.05 *$ & -0.02 & -0.02 \\
\hline Time & 0.58 & -0.00 & 0.02 & 0.03 \\
\hline Transition & 0.40 & 0.04 & -0.09 & -0.03 \\
\hline Time $\mathrm{x}$ Transition & 0.91 & $0.11 *$ & $-0.07 \dagger$ & -0.04 \\
\hline Pretask & & & & $0.27 * * *$ \\
\hline Peak & & & & $0.49 * * *$ \\
\hline Pretask x Time & & & & -0.00 \\
\hline Peak x Time & & & & 0.03 \\
\hline Pretask x Transition & & & & -0.04 \\
\hline Peak x Transition & & & & $-0.09 \dagger$ \\
\hline Pretask x Time $\mathrm{x}$ Transition & & & & $0.10^{*}$ \\
\hline Peak x Time $\mathrm{x}$ Transition & & & & 0.04 \\
\hline
\end{tabular}

Note . Speech anticipation $=$ cortisol concentration in the pre-task sample minus cortisol concentration in the recovery sample, Speech delivery $=$ peak cortisol concentration in post- 
task samples minus cortisol concentration in the pre-task sample, AUCi $=$ Area Under the Curve with respect to increase (Pruessner et al., 2003). SPM = sum score on the Standard Progressive Matrices, PDS = mean score on the pubertal development scale (Petersen et al., 1988), T1 Age = age at Time 1 centered at the age of the youngest participant, Time $=$ age at time of testing minus age at Time 1 . Transition was coded as $0=$ recursive thinking at both times, $1=$ from non-recursive thinking at Time 1 to recursive thinking at Time 2 . The analyses were performed on the natural logarithm of the cortisol concentration (nmol/l). $\dagger p<.10, * p<.05, * * p<.01, * * * p<.001$ 


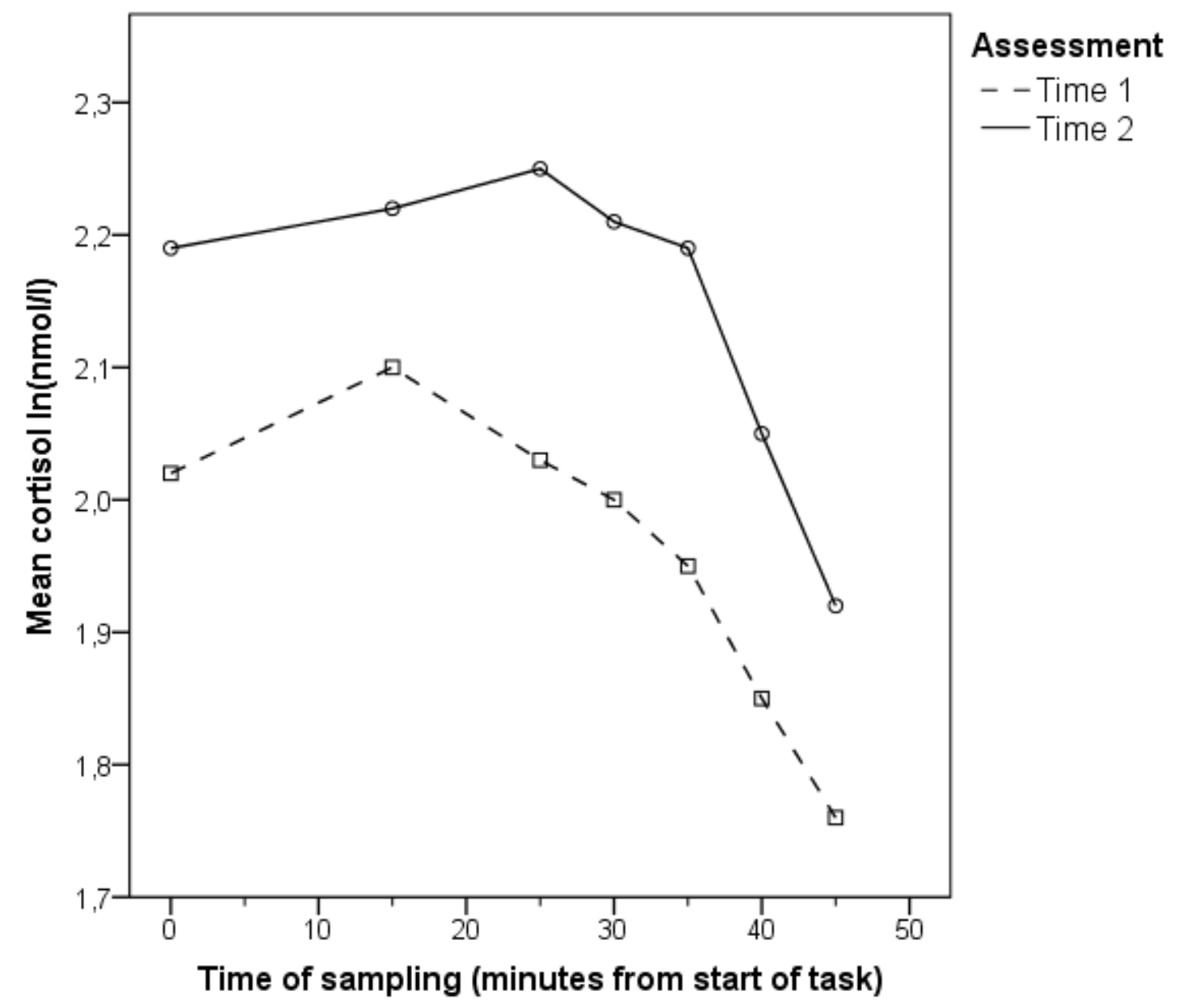

Figure 1. Mean natural logarithm of the cortisol concentration (nmol/l) for the seven saliva samples taken directly before and 15, 25, 30, 35, 40, and 45 min after the beginning of the Leiden Public Speaking Task at Time1 and Time 2. 


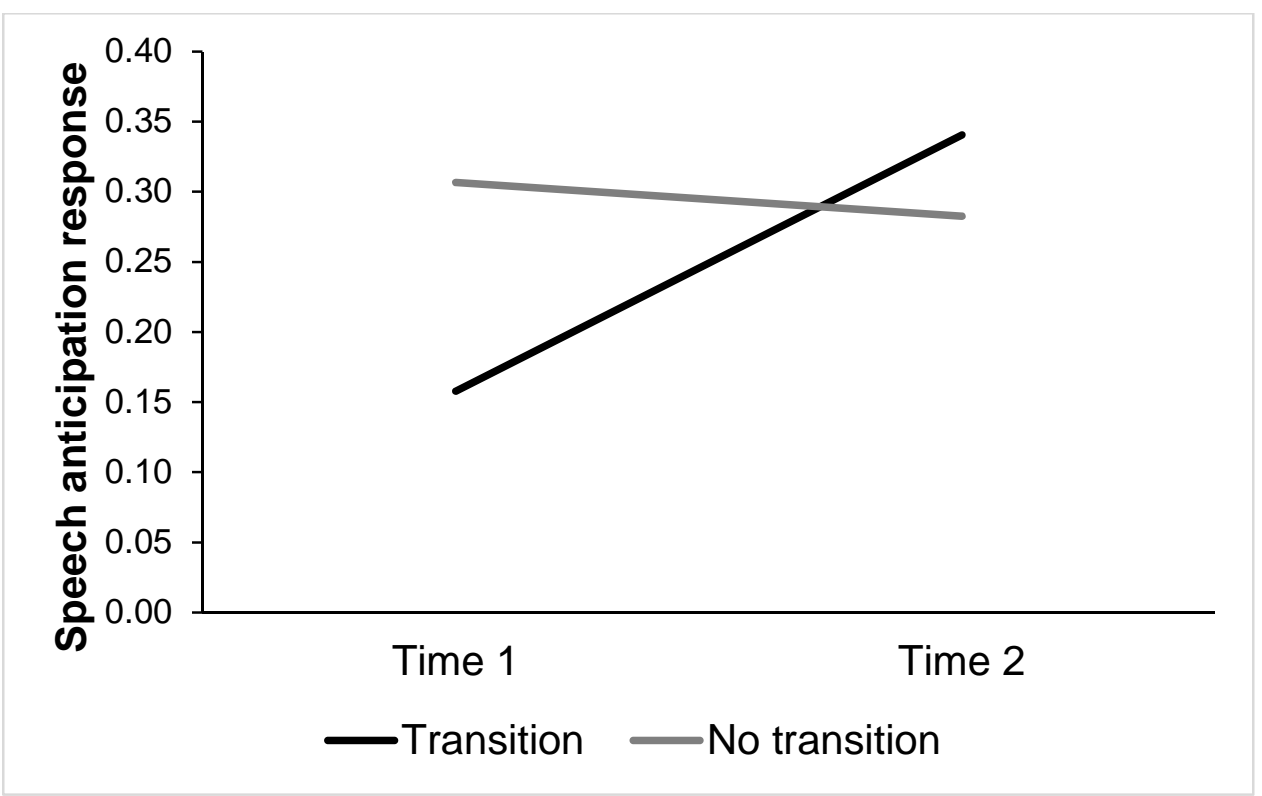

Figure 2. Predicted speech anticipation response at Time 1 and Time 2 for participants who transitioned from non-recursive thinking at Time 1 to recursive thinking at Time 2 and for participants who demonstrated recursive thinking at both times. The speech anticipation response represents the difference in the natural logarithm of the cortisol concentration $(\mathrm{nmol} / \mathrm{l})$ between the pre-task sample and the recovery sample. Control variables were set at their respective means at Time 1 and Time 2. 


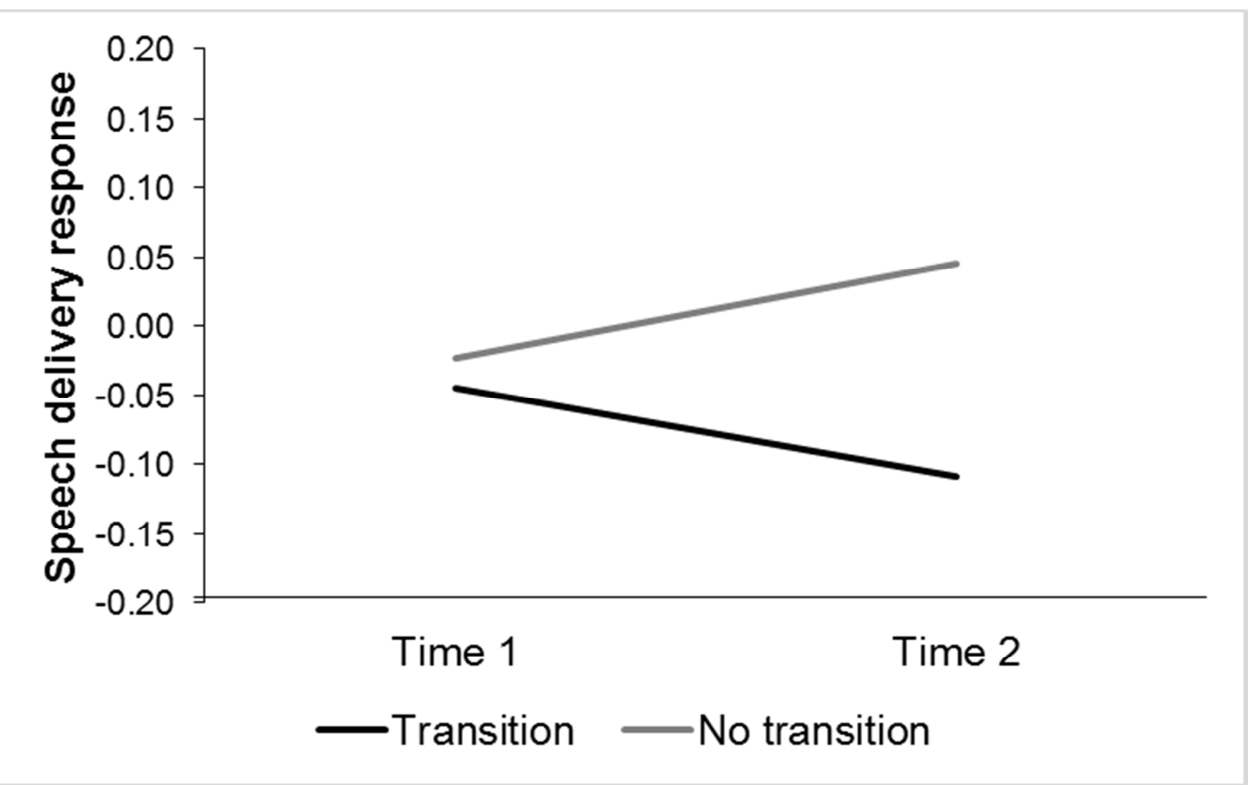

Figure 3. Predicted speech delivery response at Time 1 and Time 2 for participants who transitioned from non-recursive thinking at Time 1 to recursive thinking at Time 2 and for participants who demonstrated recursive thinking at both times. The speech delivery response represents the difference in the natural logarithm of the cortisol concentration (nmol/l) between the post-task peak sample and the pre-task sample. Control variables were set at their respective means at Time 1 and Time 2 . 


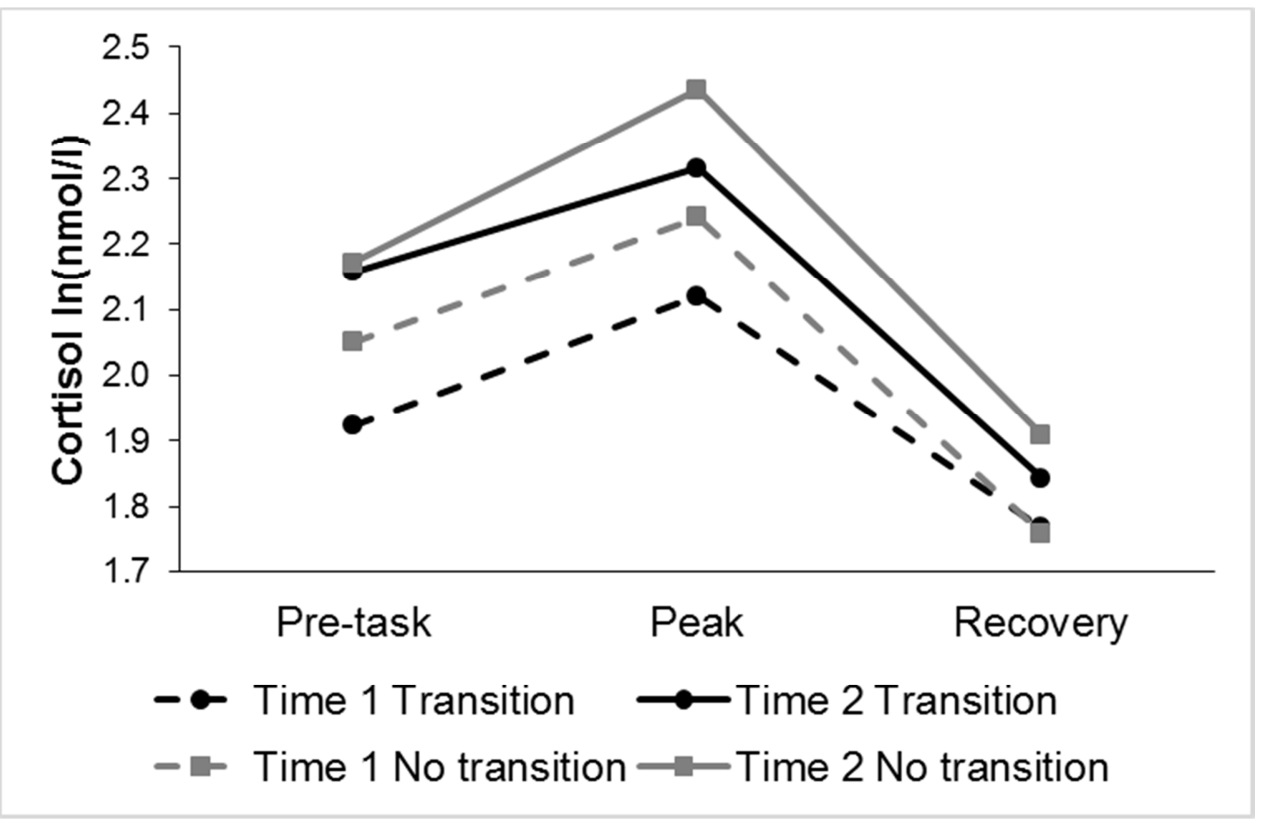

Figure 4. Predicted natural logarithm of the cortisol concentration in pre-task, post-task peak and recovery samples at Time 1 and Time 2 for participants who transitioned from nonrecursive thinking at Time 1 to recursive thinking at Time 2 and for participants who demonstrated recursive thinking at both times. Control variables were set at their respective means at Time 1 and Time 2. 


\section{Appendix. Items of the Recursive Thinking Test Used in the Present Study}

\section{Action items}

1. The boy is thinking that the girl is talking to the (male) teacher.

2. The boy is thinking that he is talking to the girl.

3. The boy is thinking that the girl is talking to him.

\section{One-loop recursion items}

4. The boy is thinking that the girl is thinking of the (male) teacher.

5. The boy is thinking that he is thinking of the girl.

6. The boy is thinking that the girl is thinking of him.

7. The boy is thinking that the girl is thinking of herself.

8. The boy is thinking that he is thinking of himself.

\section{Two-loop recursion items}

9. The boy is thinking that the girl is thinking of the (male) teacher thinking of the (female) teacher.

10. The boy is thinking that he is thinking of the girl thinking of herself.

11. The boy is thinking that the girl is thinking of him thinking of her.

12. The boy is thinking that he is thinking of himself thinking of himself.

13. The boy is thinking that the girl is thinking of him thinking of himself.

14. The boy is thinking that he is thinking that the girl is thinking of him. 


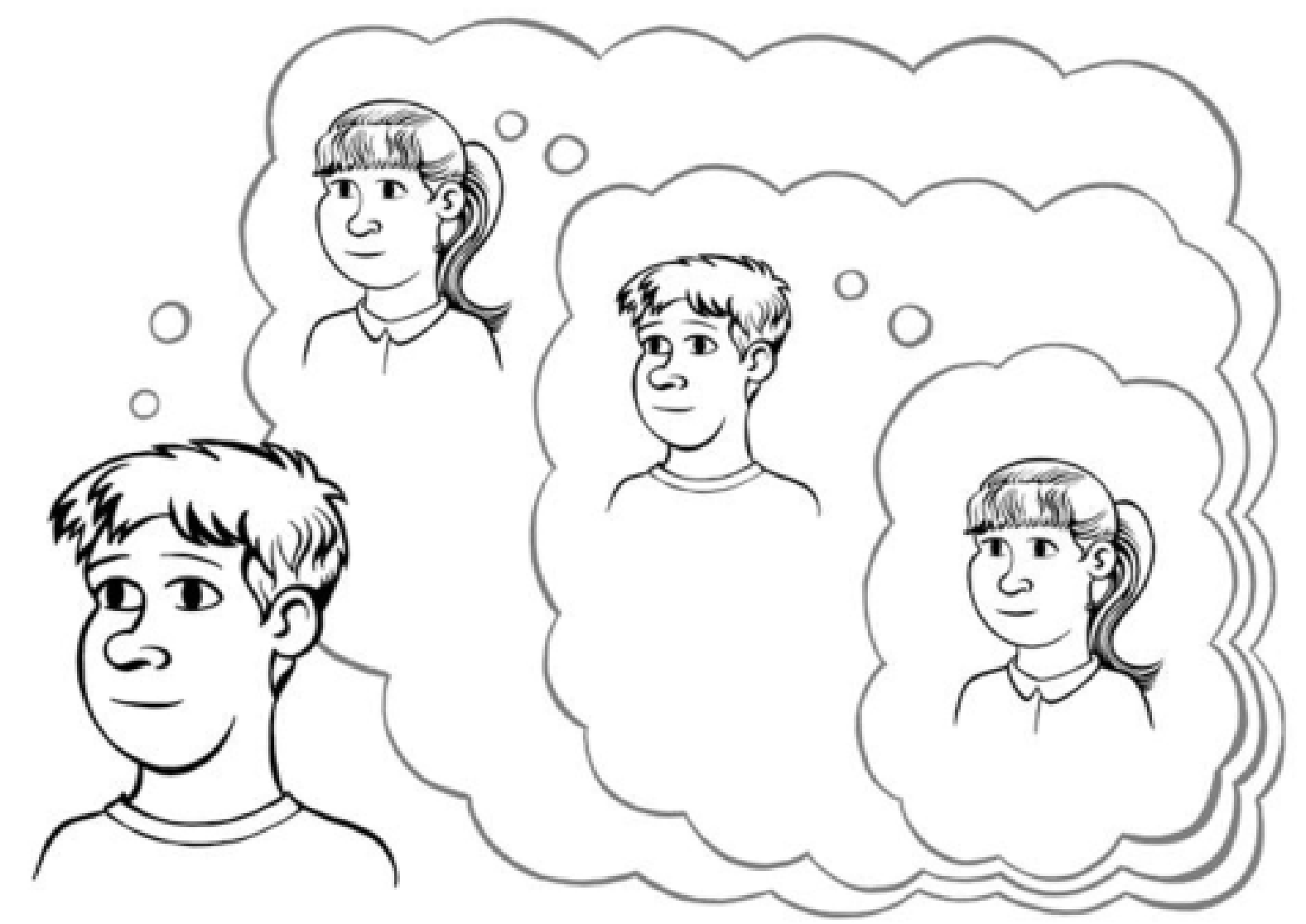

Figure A1. Cartoon of item 11: The boy is thinking that the girl is thinking of him thinking of her. Copyright John Miers. 